\title{
El gluten del trigo y su rol en la industria de la panificación
}

\author{
Rafael Villanueva Flores \\ Universidad de Lima. Lima, Perú \\ Correo electrónico: Rvillan@correo.ulima.edu.pe
}

Recibido: 25 de marzo de 2014 / Aprobado: 6 de mayo de 2014

RESUMEN: El gluten es la proteína del trigo que le confiere a la harina propiedades únicas para obtener una masa viscoelástica y cohesiva capaz de retener gas y preparar productos horneados aireados y livianos como panes, bizcochos y galletas. No hay harina sucedánea capaz de formar una masa con propiedades viscoelásticas similares. El gluten está formado por dos proteínas: gliadina (una prolamina) y glutenina (una glutelina). Diversos modelos se han propuesto a través de los años para tratar de explicar la manera en la que los componentes del gluten interactúan, pero aún existe una brecha en el conocimiento y entendimiento de la exclusividad del gluten y su rol en la elaboración de diferentes productos horneados.

Palabras clave: cereales / trigo / gluten / panificación

\section{Wheat gluten and its importance in the baking industry}

ABstract: Gluten is the wheat protein that gives flour unique viscoelastic, cohesiveness and gas holding capacity properties, fundamental aspects in the production of aerated and low weight baked products like breads, cakes and crackers. No other flour forms a dough with similar viscoelastic character. Gluten is composed of gliadin (a prolamine) and glutenin (a gluteline). Over the years, different models have been proposed to explain the way gluten components interact, but there is still a gap in knowledge and understanding of gluten uniqueness and its role in the production of baked products.

Key words: cereals / wheat / gluten / baked products 


\section{INTRODUCCIÓN}

El trigo es uno de los alimentos básicos más importantes del mundo. Anualmente se procesan más de 600 millones de toneladas para consumo humano. En ningún otro alimento básico influyen tanto las oscilaciones de las características de la materia prima en la naturaleza y las propiedades de procesamiento como en la harina de trigo. Factores como la clase de cereal, la calidad del suelo, el clima, las condiciones de cosecha, el almacenamiento y la molienda causan diferencias en las condiciones de calidad que provocan perturbaciones en el procesamiento, rendimiento y características de los productos finales horneados.

Entre los cereales, solo la harina de trigo tiene la capacidad de formar una masa resistente, viscoelástica y cohesiva capaz de retener gas y producir productos horneados ligeros y aireados (Hoseney, 1994). Este es el motivo por el cual el pan y los bizcochos crecen o levantan en el horno y no "caen". Las proteínas del trigo —y más específicamente el gluten-son las responsables de esta característica única del trigo, aunque la manera precisa en la que los componentes interactúan en el gluten para lograr estas propiedades únicas aún está sin resolver.

Diversos trabajos de investigación en torno al gluten se han llevado - y se están llevando - a cabo para identificar los factores que definen la calidad de un trigo para un determinado uso final. El presente trabajo de investigación presenta un resumen de los avances en el conocimiento del gluten.

\section{HISTORIA DEL GLUTEN}

Aunque el trigo se consume desde hace miles de años, el descubrimiento del gluten es relativamente reciente. Jacopo Bartholomew Beccari, un profesor de Química de la Universidad de Boloña, descubrió el gluten en 1728, cuando lo aisló lavando a mano una masa de harina. Lo describió como un material gelatinoso opuesto a cualquier material soluble amiláceo. Igualmente, observó que este material gelatinoso, una vez aislado, no era capaz de ser mezclado más con agua y tenía propiedades físicas únicas (Beach, 1961). Este descubrimiento fue significativo porque hasta ese momento se pensaba que las proteínas eran solo de origen animal.

El siguiente paso en la investigación del gluten ocurrió en 1823, cuando Osborne y Voorhees desarrollaron el procedimiento de fraccio- 
namiento hasta hoy utilizado. Las proteínas del gluten fueron separadas en cuatro fracciones sobre la base de diferencias en solubilidad en el sistema clásico de cuatro solventes: agua, sal diluida, 70 \% etanol y ácido/álcali diluidos, que corresponden a albuminas, globulinas, prolaminas y glutelinas, respectivamente (Osborne \& Voorhees, 1893). Ninguna de las proteínas individualmente podía ser clasificada correctamente como gluten. Sin embargo, la combinación de prolamina y glutelina produjeron el producto descubierto por Beccari dos centurias antes. Osborne (1907) describió sus componentes proteicos como fracción gliadina monomérica y fracción glutenina polimérica muy agregada.

El tercer descubrimiento mayor, que cambió el entendimiento del gluten, fue la publicación de Balls y Hale (1936), en la que se describió el desdoblamiento del gluten después de la adición de agentes reductores. Los autores examinaron la pérdida de integridad del gluten en presencia de compuestos como cisteína y glutatión. No fueron capaces de identificar el fenómeno y pensaron que podía estar relacionado con la activación de una enzima que trozaba los enlaces disulfuros. El hallazgo inadvertido fue el reconocimiento de que los enlaces disulfuros son necesarios para la estructura y funcionalidad del gluten.

Estos tres descubrimientos claves ocurrieron en tres siglos diferentes y fueron vitales para el entendimiento inicial del gluten del trigo. Este ritmo de investigación fue acelerado con el paso del tiempo hasta la actualidad. Sin embargo, a pesar de toda la investigación realizada en el gluten del trigo, estamos todavía lejos del entendimiento completo de estas proteínas.

\section{COMPOSICIÓN DEL GLUTEN}

El gluten del trigo contiene alrededor de $80 \%$ de proteínas, 5 a $10 \%$ de lípidos, almidón residual, carbohidratos y proteínas insolubles en agua entrapadas en la masa (Nierle y El Baya, 1990). Está compuesto de dos clases principales de proteínas: gliadina (una prolamina) y glutenina (una glutelina).

Las gliadinas son un grupo de proteínas con propiedades similares. Se caracterizan por su solubilidad en carbinoles alifáticos inferiores, especialmente etanol, y en algunos carbinoles aromáticos como el fenol. Se les conoce como proteínas solubles en alcohol. Tienen un peso molecular promedio de 40,000, son de cadena simple y extremadamente 
pegajosas cuando se hidratan. Además, muestran poca o ninguna resistencia a la extensión y parecen ser las responsables de la cohesión de la masa (Hoseney, 1994). Presentan un polimorfismo intervarietal muy marcado y sus patrones electroforéticos no son afectados por condiciones ambientales (Zillman \& Bushuk, 1979).

Las gluteninas son un grupo heterogéneo de proteínas. Se caracterizan por su solubilidad en ácidos y álcalis diluidos. Son de cadena múltiple y peso molecular variable (de 10,000 a varios millones) que comprende a gluteninas de bajo y elevado peso molecular; resiente y gomosa, pero propensa a la ruptura. Las gluteninas, aparentemente, proporcionan a la masa la propiedad de resistencia a la extensión (Hoseney, 1994). Estudios de reconstitución han indicado que para una cosecha de una variedad en particular de trigo, la cantidad relativa de glutenina está directamente relacionada con la resistencia a la extensión de la masa (MacRitchie, 1980, 1987). Sin embargo, debido a la solubilidad inconsistente de las fracciones de gliadina y glutenina, y a la superposición de sus componentes, resulta difícil demostrar esta relación para diferentes variedades y cosechas.

Finney y Barmore (1948) mostraron que las proteínas del gluten eran las responsables de las diferencias en las propiedades de panificación de harinas de trigo de diferentes calidades panaderas. También se acepta que el volumen del pan está correlacionado con el contenido de proteína de la harina. Sin embargo, no hay consenso acerca de qué aspectos de las proteínas son los responsables por estas diferencias. La relación glutenina/gliadina en el gluten ha sido propuesta como un factor importante, pero no hay evidencia experimental que lo demuestre (MacRitchie, 1980). El contenido de tiol y disulfuro también han sido sugeridos como variables importantes, pero no hay correlación analítica establecida (Bloksma, 1975; Graveland et al., 1978). Los estudios de la composición de aminoácido, movilidad electroforética y distribución de peso molecular tampoco indican correlación alguna (McDonald \& Gilles, 1967).

La disponibilidad de nitrógeno durante el crecimiento del trigo es un factor que determina el rendimiento de la cosecha, las proteínas del gluten y la calidad del grano (Gianibelli \& Sarandon, 1990). El contenido de proteína del trigo depende en gran medida de factores agronómicos y ambientales como el nitrógeno y la humedad del suelo, y la temperatura durante las etapas de crecimiento (Bushuk, 1985). 
La genética de la variedad del trigo es la que determina qué proteínas están presentes y la proporción relativa entre ellas (Hoseney, 1994). En el trigo existe una relación inversa entre contenido de proteína y rendimiento (Loffler et al., 1985).

Los trigos comunes (duros y suaves) son hexaploises, es decir, contienen tres genomas $\mathrm{A}, \mathrm{B}$ y $\mathrm{D}$, mientras que el trigo durum es tetraploide y contiene solo genomas A y B. Los científicos han encontrado la localización en el cromosoma de algunos de los genes para proteínas y polipéptidos específicos (Hoseney, 1994).

\section{PROTEÍNAS DEL GLUTEN}

Las proteínas del gluten son las proteínas de almacenamiento del trigo. Son fáciles de aislar porque son insolubles en agua. Tienen elevado contenido de ácido glutámico y prolina, bajo contenido de lisina y baja densidad de carga (Hoseney, 1994).

El gluten sirve como fuente primaria de carbono, nitrógeno y - en menor medida - azufre de la planta inmadura. La glutamina es el aminoácido más abundante y, junto con la prolamina y la glicina, conforma más del 50 \% de los aminoácidos residuales en el gluten (Hoseney, 1994). Esto puede limitar los tipos de conformaciones estructurales secundarias que son posibles para el gluten, especialmente si los tres aminoácidos tienden a ser localizados en posiciones conservadas en motivos repetitivos. Específicamente, el tipo II $B(i \rightarrow \mathrm{i} \pm 3$ enlaces de hidrógeno) y en menor grado el tipo I $B$ son las conformaciones más probables cuando una x-prolina-glicina-x es abundante (Horton et al., 2006).

Otras características adicionales del gluten son el número relativamente elevado de aminoácidos hidrofóbicos residuales (aproximadamente $35 \%$ ) y la baja densidad de carga debido a la relativa carencia de aminoácidos básicos y a la presencia de la forma amida de los aminoácidos ácidos. Metionionina, triptófano y lisina son los aminoácidos limitantes en el gluten (Hoseney, 1994).

El procedimiento de fraccionamiento de Osborne fue un paso adelante en la identificación de los dos grupos principales de proteínas que comprenden el gluten. El desarrollo de la electroforesis condujo a este descubrimiento y la consecuente aclaración de que las gliadinas y gluteninas son dos grupos amplios de proteínas, cada una compuesta de muchas subunidades individuales. 
Este primer estudio controlado de electroforesis para demostrar que el gluten del trigo está compuesto por más de dos proteínas distintas fue realizado en 1959 (Jones et al., 1959). Dos fracciones fueron atribuidas tentativamente a la glutenina y cuatro a la gliadina utilizando electroforesis de frontera en movimiento. Woychik et al. (1961) fueron los primeros en fraccionar claramente la gliadina en subunidades individuales. Ellos reportaron la presencia de $\alpha-,, b-, \gamma-y$ $\omega$-gliadinas con subunidades adicionales en las a y $B$ gliadinas $\left(\alpha_{1-2} y\right.$ $B_{1-4}$, respectivamente).

Desde la conclusión de esta investigación, sabemos que las cuatro fracciones ( $\alpha$, B, y y $\omega$ ) tienen peso molecular entre 15 y 60 kilos Dalton y representan el 50\% de las proteínas del gluten (Lásztity, 1996). A las gliadinas $a, B$ y $\gamma$ se les consideran ricas en azufre (23-35 Cys + residuos met / 100000 g de proteína) y generalmente se encuentran en el rango de 25 a 40 kilos Dalton (Eliasson y Larsson, 1993). Se asume que todas las gliadinas existen como monómeros globulares en estado natural debido a su baja densidad de carga y a enlaces disulfuros intramoleculares. La prolina, glicina y residuos de glutamina, que comprenden la mayoría de aminoácidos, se distribuyen de una manera que resulta generalmente en una estructura aperiódica; sin embargo, corridas cortas de una estructura a helicoidal están disponibles para formarse en áreas pobres en estos tres aminoácidos, que resulta una estructura que puede llegar a ser 33 \% a helicoidal (Lásztity, 1996).

Jones et al. (1959) identificaron primero dos fracciones de glutenina y cuatro fracciones de gliadina utilizando electroforesis de frontera en movimiento, mientras que Woychik et al. (1961) identificaron solamente una banda de glutenina utilizando electroforesis en gel. Por su parte, Nielsen et al. (1962) examinaron más la heterogeneidad del gluten dividiendo los enlaces disulfuros con ácido perfómico. El análisis de sedimentación demostró que la glutenina es un sistema polidisperso con masa molecular promedio entre 35 y 150 kilos Dalton.

Desde entonces, las investigaciones han demostrado que las dos fracciones principales de la glutenina son subunidades de bajo peso molecular y subunidades de alto peso molecular. El peso molecular estimado para los complejos poliméricos de la glutenina varía entre 100 y más de 10 millones (Wieser et al., 2006). El peso molecular estimado de las subunidades de alto peso molecular varía entre 70 y 136 , y para las subunidades de bajo peso molecular, entre 20 y 45 luego de la reducción de enlaces disulfuros intermoleculares (D’Ovidio \& Macsi, 2004). 


\section{MODELOS DEL GLUTEN}

La interacción entre gliadina y glutenina en la presencia de agua y energía mecánica es la que resulta en la formación del gluten. La glutenina y la gliadina proporcionan diferentes propiedades reológicas a la masa luego de haberse formado una estructura dinámica. Se estima que la gliadina contribuye a la extensibilidad, mientras que la glutenina contribuye a la elasticidad. En el contexto de una masa, especialmente la de pan, se dice que el gluten forma una red continua y que esta red es la responsable por las propiedades viscoelásticas únicas del gluten. Diversos modelos han sido propuestos a través de los años para describir las bases de la red del gluten que le permiten tener estas características reológicas únicas.

Ewart $(1968,1972,1977)$ propuso que el enlace aleatorio de las gluteninas por enlaces disulfuros intercatenarios, cabeza-cola, definía la reología de la red del gluten, especialmente su elasticidad. El modelo, sin embargo, no indicaba un rol específico de las gliadinas. No obstante, el reconocimiento de que la glutenina polimérica es la responsable clave de la naturaleza continua de la red y sus propiedades elásticas han impulsado a comprender mejor su comportamiento.

Kasarda et al. (1976) propusieron que las gluteninas formaban únicamente enlaces disulfuros intercatenarios y las interacciones no covalentes entre moléculas conducían a la creación de la red de gluten. Estas interacciones no covalentes son dependientes, sin embargo, en la formación de conformaciones estructurales específicas por las gluteninas sobre la formación de enlaces disulfuros intramoleculares.

Khan y Bushuk (1979) propusieron una teoría híbrida en la cual la glutenina era capaz de formar enlaces disulfuros inter- e intramoleculares. Identificaron dos fracciones de gluten: una de bajo peso molecular, que forma enlaces no covalentes fuertes, y otra de elevado peso molecular, que forma enlaces disulfuros. Los enlaces disulfuros intermoleculares permiten el desarrollo de una red polimérica, mientras que la estabilidad requerida para interacciones no covalentes es proporcionada por las gluteninas disulfuras enlazadas intramolecularmente.

Graveland et al. (1985) propusieron uno de los modelos finales que precedió al modelo actualmente aceptado. En este modelo, las gluteninas forman un polímero muy estructurado altamente ramificado y con asociaciones específicas no aleatorias. Las gluteninas fueron propuestas como participantes de enlaces disulfuros inter- o intramoleculares 
con una cadena principal que forma los enlaces disulfuros lineales cabeza-cola, como lo propuso Ewart (1968, 1972, 1977). Adicionalmente, se planteó que las subunidades de gluteninas con enlaces disulfuros intramoleculares forman ramas en la cadena principal mediante enlaces intermoleculares adicionales.

Todos los modelos precedentes tratan exclusivamente del rol de la glutenina y los enlaces disulfuros cruzados en la red del gluten. El modelo actual aceptado fue descrito por Shewry et al. (2001). En este modelo las subunidades de elevado peso molecular forman la columna vertebral mediante enlaces disulfuros cruzados cabeza-cola, con pequeño grado de enlaces laterales disulfuros entre las cadenas de subunidades de elevado peso molecular. Las subunidades de bajo peso molecular también participan sirviendo como conectores de cadena o terminadores dependiendo del número (par o impar) de grupos sulfidrilos disponibles para enlaces disulfuros. Las gliadinas interactúan no covalentemente con cadenas de subunidades de elevado peso molecular, cumpliendo un rol modulador en términos de la elasticidad de la glutenina.

$\mathrm{Al}$ revisar los modelos de organización de la red del gluten se obtiene una buena visión sobre las propiedades reológicas únicas de una masa de harina de trigo. Otras teorías propuestas refinan el modelo actualmente aceptado. Ellas describen la formación de la red de gluten y las interacciones entre gliadinas y gluteninas que dan lugar al comportamiento reológico de una masa.

La asunción implícita de Belton (1999) de la teoría del lazo de cola para el desarrollo de una masa de harina de trigo es que las estructuras secundarias hoja $B$ (colas) son inherentemente menos elásticas que las estructuras secundarias de giro $B$ (lazos) en una proteína de gluten. Como resultado, las bases para el aumento del torque y tensión de endurecimiento observado durante el mezclado representa una transición de estructuras secundarias de giro $B$ a hoja $B$ en el gluten. El estiramiento progresivo plegado de la red de gluten con enlaces cruzados durante el mezclado eventualmente resulta en un ratio entre estructuras de giro $B$ a hoja $B$ resistentes a la deformación. El mezclado por encima de este punto resulta en la ruptura de la red del gluten y como consecuencia disminuyen el torque de mezclado y las medidas de endurecimiento.

Hammer y Van Vliet (2000) describieron el desarrollo de una masa como la hiperagregación de polímeros de glutenina. Los tres estados de 
agregación, como originalmente se plantearon, incluían: 1) la formación enlaces cruzados disulfuros entre las subunidades de glutenina en el grano de trigo; 2) agregación de polímeros de glutenina y enlaces de hidrógeno en el grano de trigo en etapas tempranas de mezclado; y 3) supraagregación de partículas por posteriores enredos resultante de mezclado tardío y durante el procesamiento. Esto se basa en que la glutenina existe como una macropartícula, referida como macropolímero de glutenina (MPG), y que la red de gluten en una masa se forma por agregación de productos descompuestos de MPG.

Singh y MacRitchie (2001) postularon que el desarrollo de una masa se explica mejor en el contexto de un sistema de un polímero entrelazado. La propuesta se basa en la teoría físico-química y de polímeros para describir las diversas propiedades del gluten y de la masa. En la masa, el gluten se comporta como un polímero entrelazado mediante enlaces disulfuros y enredos inter- e intramoleculares. El mezclado y la hidratación dan como resultado el aumento segmentado de la movilidad parcial del gluten, lo cual fomenta la formación de entre enlaces de un umbral de tamaño crítico. A este umbral de tamaño de polímero, el mezclado adicional produce la ruptura de la red de entrelazamiento. Es la formación de la red de entrelazamiento la que da lugar a las curvas típicas de torque registradas para el mezclado de una masa de harina de trigo. Singh y MacRitchie (2001) describieron las propiedades elásticas de la masa en términos de desplazamiento en equilibrio, es decir, al aplicar a la red gluten una fuerza de estiramiento da como resultado el desplazamiento y despliegue de las proteínas del gluten del equilibrio, y el retiro de la fuerza ocasiona la recuperación del estado de equilibrio en la medida en que alguna obstrucción viscosa de la gliadina y nuevos enredos lo permitan.

Fenómenos adicionales, que están muy bien caracterizados por la teoría de entrelazamiento, incluyen velocidad de mezclado, sobremezclado, solubilidad del gluten, dureza del grano, resistencia a la extensión y extensibilidad de la masa y funcionalidad del gluten modificado.

\section{NUEVOS CONCEPTOS DEL GLUTEN}

Un factor clave lo constituye el hecho de que todos los trabajos previos en la formación y función de la red de gluten están basados inherentemente en el pan como modelo "ideal" y la calidad del gluten se describe típicamente en términos de su funcionalidad en el pan. Dentro del 
contexto de la diversidad de productos hechos con harina de trigo, la pregunta que surge es si el modelo se aplica a todos los productos elaborados con harina de trigo. Aún existe una brecha en el conocimiento y entendimiento del gluten y su rol en productos diferentes al pan que merece atención adicional. Hay mucho que investigar, por ejemplo, en la relación entre la estructura del gluten y la funcionalidad en trigos suaves y duros.

En años recientes, la investigación sobre los trigos suaves se ha enfocado principalmente en las puroindolinas. Las diferencias en la textura del grano pueden ser atribuidas a mutaciones en puroindolinas (Morris, 2002). Los trigos suaves retienen los genes silvestres de puroindolinas que expresan estas proteínas de una manera más funcional en términos de modificación de propiedades de textura. Por otro lado, los trigos duros poseen versiones mutadas de estos genes y son de textura dura. Caso extremo es el de los trigos durum, que al carecer de estos genes tienen una textura muy dura.

Los intentos para identificar la calidad del trigo suave se han centrado principalmente en el impacto en el procesamiento y características de galletas saladas y dulces. Las conclusiones de Souza et al. (2011, 2012) indican que los rasgos genéticos, como suavidad equivalente, rendimiento de molienda y capacidad de retención de sucrosa solvente, deben ser utilizados para dirigir la selección de nuevas líneas de trigo. Lo resaltante es que las características funcionales del gluten no pueden ser manejadas tan cuidadosamente en trigos suaves como en trigos duros, quizás por los modelos que se emplean como determinantes de la calidad del producto final y por la falta de comprensión de la funcionalidad del gluten del trigo suave en comparación con la del trigo duro.

La estructura básica de los bloques de gluten, o los tipos y cantidades de gliadina y glutenina presentes están influenciados tanto por factores genéticos como por ambientales. Los trigos duros y suaves son inherentemente diferentes en el nivel genético en cuanto a los tipos de subunidades presentes. Las condiciones ambientales como el clima, las condiciones del suelo y el manejo de prácticas agronómicas también influyen en las características de la gliadina y glutenina, al igual que en la cantidad de la proteína. En conjunto, estos factores determinan la cantidad y calidad del gluten. Una vez que un lote de trigo llega al molino, sus parámetros de calidad pueden ser mezclados con los de otros trigos, con la finalidad de alcanzar ciertas especificaciones (típicamente proteína y cenizas o valores reológicos) en la harina. Desafor- 
tunadamente, estas especificaciones son una pobre indicación del comportamiento de una harina. La cantidad de proteína sirve como buena indicación de la cantidad de gluten, pero indica poco con respecto a la calidad y funcionalidad.

Estudios recientes, hechos mediante una técnica de cizallamiento, prueba de pico de gluten (PPC), para medir la cinética de agregación del gluten, demostraron la falta de información suministrada por la cantidad de proteína. Esta prueba se ha utilizado para diferenciar las propiedades de agregación (torque y tiempo) del gluten en un batido (8,5 g de harina: 9,5 g de $\mathrm{CaC}_{12}$ 0,5 M). Diferentes trigos con contenido similar de proteína mostraron diferentes valores de torque. Otro punto interesante es que hay diferencias inherentes entre las gliadinas y gluteninas de trigos duros y suaves. Se necesita mayor investigación para determinar de qué manera las fracciones específicas y subunidades de estas proteínas influyen en la funcionalidad del gluten en el procesamiento de productos a base de harina de trigo. La espectroscopia infrarroja con transformada de Fourier (EITF) ha sido utilizada como herramienta para el estudio de la estructura secundaria de las proteínas en la harina y durante el desarrollo de la masa. Li et al. (2006) reportaron un aumento en la estructura de la harina y gluten de hoja 8 como consecuencia de hidratación no mecánica, mientras que otros investigadores (Mejía et al., 2007; Robertson et al., 2006) han reportado el aumento de estructura de hoja $B$ a expensas de estructura de giro $B$ durante la deformación y mezclado de harina o de gluten.

En el contexto del mezclado de una masa, los resultados indican que las harinas de trigo suave y duro no comparten una evolución estructural similar del gluten durante el transcurso del mezclado. La harina de trigo duro muestra una evolución estructural que está en línea con lo propuesto por Belton (1999), mientras que la harina de trigo suave no muestra un cambio significativo en la estructura secundaria del gluten durante el mezclado. Estos resultados sugieren que los modelos actuales fallan en explicar adecuadamente la red de gluten en sistemas y productos. Es importante también investigar el impacto en la interacción del gluten con otros ingredientes utilizados en la formulación de productos hechos a base de harina de trigo. Trabajos de investigación conducidos por Huschka et al. (2011) han mostrado que la hidrofobicidad superficial difiere sustancialmente entre las harinas de trigo suave y duro, afectando potencialmente su habilidad para interactuar con lípidos y otros ingredientes hidrofóbicos en las formulaciones. El 
número de lugares hidrofóbicos puede variar, al igual que la afinidad con la cual este lugar es capaz de unir materiales hidrofóbicos. En el caso de harinas de trigo duro, aparentemente, hay un número menor de lugares de afinidad elevada, mientras que en harinas de trigo suave hay un gran número de lugares de baja afinidad (Huschka et al., 2011). La hidrofobicidad superficial de harina de trigos suaves y duros se desarrolla durante el proceso de mezclado.

\section{CONCLUSIONES}

Se requiere mayor investigación en los parámetros de calidad del gluten para poder definir el uso de la harina para un producto en particular. Una harina de calidad es aquella que puede desarrollar un gluten que interactúe con los ingredientes utilizados en la formulación para obtener las características y el rendimiento esperado en el producto final.

\section{REFERENCIAS}

Balls, A., \& Hale, W. (1936). Further studies on the activity of proteinase in flour. Cereal Chem., 13, 56-64.

Beach, E. (1961). Beccari of Bologna: The discoverer of vegetable protein. J. Hist. Med., 26, 354-373.

Belton, P. (1999). On the elasticity of wheat gluten. J. Cereal Sci., 29, 103-107.

Bloksma, A. (1975). Thiol and disulfide groups in dough rheology. Cereal Chem., 52; 170-183.

Bushuk, W. (1985). Flour proteins: Structure and functionality on dough and bread. Cereal Foods World, 30, 447-451.

D’Ovidio, R., \& Macsi, S. (2004). The low-molecular-weight glutenin subunits of wheat gluten. J.Cereal Sci., 39, 321-339.

Eliasson, A., \& Larsson, K. (1993). Cereal in breadmaking: a molecular colloidal approach. New York: Marcel Dekker, Inc.

Ewart, J. (1968). A hypothesis for the structure and rheology of gluten. J. Sci. Food Agric., 19, 617-623.

Ewart, J. (1972). A modified hypothesis for the structure and rheology of gluten. J. Sci. Food Agric., 23, 687-699. 
Ewart, J. (1977). Re-examination of the linear glutenin hypothesis. $J$. Sci. Food Agric., 28, 191-199.

Finney, K., \& Barmore, M. (1948). Loaf volume and protein content of hard winter and spring wheats. Cereal Chem., 25, 291-312.

Gianibelli, M., \& Sarandon, S. (1990). Effect on late nitrogen fertilization on the gluten content and technological quality of bread wheat. En W. Bushuk, \& R. Trachuk (Eds.), Gluten Proteins (pp. 755764). St. Paul, MN: AACC International.

Graveland, A., Bosveld, P., \& Marseille, J. (1978). Determination of thiol groups and dsisulfide bonds in wheat flour and dough. $J$. Sci. Food Agr., 29, 53-61.

Graveland, A., Bosveld, P., Lichtendonk, W., Marseille, J. P., Moonnen, J., \& Scheepstra, A. (1985). A model for the molecular structure of the glutenins from wheat flour. J. Cereal Sci., 3, 1-16.

Greene, F. \& Anderson, O. (1990). Predictive analysis of wheat high molecular weight glutenin subunit secondary structure: Environments of the cysteines. En W. Bushuk, \& R. Trachuk (Eds.), Gluten Proteins 1990 (pp. 362-375). St. Paul, MN: AACC International.

Hammer, R., \& Van, T. (2000). Understanding the structure and properties of gluten: An overview. En P. R. Shewry, \& A. S. Tathan (Eds.), Wheat Gluten-Proceeding of the 7th International Workshop Gluten (pp. 125-131). Cambridge, UK: Royal Society of Chemistry

Horton, H., Moran, L. A., Scrimgeour, K., Perry, M., \& Rawn, J. (2006). Proteins: Three-dimensional structure and function. En: Principles of biochemistry (pp. 84-128). Upper Saddle River, NJ: Pearson Education Inc.

Hoseney, R. (1994). Principles of cereal science and technology. St. Paul, MN: AACC International.

Huschka, B., Bonomi, F., Marengo, M., Miriani, M., \& Seetharaman, K. (2011). Comparison of lipid effects on structural features of hard and soft wheat flour proteins assessed by front-face fluorescence. Food Chem., 133, 1011-1016.

Jones, R., Taylor, N., \& Senti, F. (1959). Electrophoresis and fractionation of wheat gluten, Arch. Biochem. Biophys., 64, 263-267. 
Kasarda, D., Bernardin, J., \& Nimmo, C. (1976). Wheat proteins. En Y. Pomeranz (Ed.), Advances in Cereal Science and Technology (pp. 158-235). St. Paul, MN: AACC International.

Khan, K., \& Bushuk, W. (1979). Studies of glutenin. XII. Comparison by sodium dodecyl sulfate-polyacrylamide gel electrophoresis of unreduced and reduced glutenin from various isolation and purification procedures. Cereal Chem., 56, 63-67.

Lásztity, R. (1996). Wheat proteins. En: The Chemistry of Cereal Proteins (pp. 19-138). Boca Raton, FL: CRC Press, Inc.

Li, W., Dobraszczyk, B., Dias, A., \& Gil, A. (2006). Polymer conformation structure of wheat proteins and gluten sub fractions revealed by ATR-FITR. Cereal Chem., 83, 407-410.

Loffler, C., Rauch, T., \& Busch, R. H. (1985). Grain and plant protein relationships in hard red spring wheat. Crop Sci., 25, 521-524.

MacRitchie, F. (1980). Physical-chemical aspects of some problems in wheat research in: Advances in Cereal Science and Technology. En: Y. Pomeranz (Ed.). III. St. Paul, MN: AACC International.

MacRitchie, F. (1987). Evaluation of contributions from wheat protein fractions to dough mixing and breadmaking. J. Cereal Sci., 6, 259-268.

McDonald, C., \& Gilles, K. (1967). Amino acid composition of wheat as relates to quality. The Baker's Dig. 1(1), 45-48.

Mejia, C., Maurer, L., \& Hamaker, B. (2007). Similarities and differences in secondary structure of viscoelastic polymers of maize a-zein and wheat gluten proteins. J.Cereal Sci. 45; 353-359.

Morris, C. (2002). Puroindolines: The molecular genetic basis of wheat grain hardness. Plant Mol. Biol., 48, 633-647.

Nielsen, H., Babcock, G., \& Senti, F. (1962). Molecular weight studies on glutenin before and after disulfide-bond splitting. Arch. Biochem. Biophys., 96, 252-258.

Nierle, W., \& El Baya, A. (1990). Functionality of modified gluten in baking. En W. Bushuk, \& R. Trachuk (Eds.), Gluten Proteins 1990 (pp. 42-46). St. Paul, MN: AACC International. 
Osborne, T., \& Voorhees, G. (1893). The proteids of wheat kernel. Am. Chem. J., 15, 392-471.

Osborne, T. (1907). The proteins of the wheat kernel. Washington, DC: Carnegie Institute of Washington.

Payne, P., Nightingale, M., Ktattinger, A., \& Holt, L. (1987). The relationship between HMW glutenin subunits composition and bread making quality of British grown wheat varieties. J. Sci. Fd. Agric., 40, 51-65.

Posner, E. (2000). Wheat. En: K. Kulp, \& J. G. Ponte (Eds.), Handbook of Cereal Science and Technology (pp. 1-29). New York: Marcel Dekker, Inc.

Reddy, P., \& Apples, R. (1990). Structure and expression of genes coding for the glutenin and gliadin proteins in wheat. En W. Bushuk, \& R. Trachuk (Ed.), Gluten Proteins 1990 (pp. 520-526). St. Paul, MN: AACC International.

Robertson, G., Gregorski, K., \& Cao, T. (2006). Changes in secondary protein structures during mixing development of high absorption (90\%) flour and water mixtures. Cereal Chem., 83, 136-142.

Shewry, P., Popineau, Y., Lafiandra, D., \& Belton, P. (2001). Findings of the Eurowheat project. Trends Food Sci. Technol., 11, 433-441.

Singh, H., \& MacRitchie, F. (2001). Application of polymer science to properties of wheat gluten. J. Cereal Sci., 33, 231-243.

Suoza, E. J., Guttieri, M. J., \& Sneller, C. (2011). Selecting sift wheat genotypes for whole grain cookies. Crop Sci., 51, 189-197.

Suoza, E., Sneller, C., Guttieri, M., Sturbaum, A., Griffey, C., Sorells, M., Ohm, H., \& Van Sanford, D. (2012). Basis for selecting soft wheat for end-use quality. Crop Sci., 52, 21-31.

Sullivan, B., Howe, M., Schmalz, F., \& Astleford.G. (1940). The action of oxidizing and reducing agents in flour. Cereal Chem., 17, 507-528.

Wieser, H., Bushuk, W., \& MacRitchie, F. (2006). The polymeric glutenins. En C. Wrigley, F. Békés, \& W. Bushuk (Eds.), Gliadin and glutenin: the perfect balance of wheat quality (pp. 213-240). St. Paul, MN: AACC International. 
Woychik, J., Boundy, J., \& Dimler, R. P. (1961). Starch gel electrophoresis of wheat gluten proteins with concentrated urea. Arch. Biochem. Biophys., 94, 477-482.

Zillman, R., \& Bushuk, W. (1979). Wheat cultivar identification by gliadin electrophoregrams. II. Effects of environmental and experimental factors on the gliadin electrophoregram. Can. J. Plant Sci., 59, 281-286. 